\title{
Polymalformative genetic syndrome with increased risk of developing cancer
}

INSERM

\section{Source}

INSERM. (1999). Orphanet: an online rare disease and orphan drug data base.

Polymalformative genetic syndrome with increased risk of developing cancer.

ORPHA:183422

Polymalformative genetic syndrome with increased risk of developing cancer (PGSIRC)

comprises a wide range of syndromes characterized by congenital malformations with a high risk of developing tumors including up to 50 different rare diseases. 